\title{
Joseph d'Arimathie, empreinte biblique des romans du Graal
}

\author{
Maïté Sauvêtre*
}

\section{José de Arimateia: impressão bíblica dos romances do Graal}

\section{Resumo}

Segundo os Evangelhos, José de Arimateia enterrou o corpo de Jesus. No século XIII, ele se tornou o guardião do Graal, um vaso com o Sangue Sagrado. Assim, a personagem torna possível vincular a Bíblia aos romances do Graal. Qual linhagem bíblica essas obras fictícias assumem? A figura bíblica revela uma pretensão de continuar e adaptar textos das escrituras de acordo com as mentalidades medievais em mudança. Graças a vários processos analógicos, o status do decurião novotestamentário é atualizado no universo graaliano. José se torna um cavaleiro santo. Os autores não se emancipam de suas fontes, mas, após os primeiros tempos cristãos, mostram como um homem pode realizar a vontade divina, levando em consideração as realidades socioculturais de seu tempo.

Palavras-chave: caráter biblico, romances do Graal, exegese.

\section{Joseph d'Arimathie, empreinte biblique des romans du Graal}

\section{Résumé}

Selon les Évangiles, Joseph d'Arimathie met le corps de Jésus au Tombeau. Au XIII ${ }^{\mathrm{e}}$ siècle, il devient le gardien du Graal, vase dans lequel il aurait recueilli le Saint-Sang. Le personnage permet ainsi de lier la Bible aux romans du Graal. Quelle filiation biblique ces œuvres fictives assument-elles alors ? La figure biblique révèle une prétention à continuer et à adapter les textes scripturaires selon des mentalités médiévales en mutation. À la faveur de différents procédés analogiques, le statut du décurion néotestamentaire s'actualise dans l'univers graalien. Joseph devient un saint chevalier. Les auteurs ne s'émancipent pas de leurs sources mais montrent comment, après les premiers temps chrétiens, un homme peut accomplir les volontés divines tout en tenant compte des réalités socioculturelles de son époque.

Mots-clés : Personnage biblique, romans du Graal, exégèse.

* Docteure ès Lettres, Sorbonne Université. Email: maite.sauvetre@gmail.com 


\title{
Joseph of Arimathea, biblical imprint in the Grail Romances
}

\begin{abstract}
According to the Gospels, Joseph of Arimathea burried Jesus Christ. During the XIII $^{\text {th }}$ century, literary tradition made him the guardian of the Holy Grail, a vase that received the Sacred Blood. Then, the Bible is linked to the Grail Romances. What biblical connections do these fictional works allow? The biblical figure of Joseph shows a desire to carry on and adapt the scriptures in harmony with changing medieval mentalities. Thanks to several analogical methods, the status of the New Testament Decurion evolves and gets renewed in the Grail Romances: Joseph becomes a holy knight. Authors do not divert from the sources but show how, after the first Christian centuries, a man can deliver the Divine will, while taking into account medieval realities.
\end{abstract}

Keywords: Biblical character, Grail Romances, exegesis.

La Bible est « l'affaire de tous» (LOBRICHON, 2008, p. 29) au Moyen Âge. Au fondement des mentalités chrétiennes, elle « a servi de lieu d'identité, fut à la source des connaissances mises en œuvres, intervint au cœur même des processus de la création littéraire »(LOBRICHON, 2003, p. 28). Elle offre un cadre à l'imaginaire. Les écrivains y voient un modèle non seulement pour la " narration » mais aussi pour "l'imagerie » (FRYE, 1984, p. 25) de leurs œuvres ${ }^{1}$. Cette filiation est alors plus ou moins assumée par les auteurs ${ }^{2}$. Au XIII ${ }^{\mathrm{e}}$ siècle, les romans du $\mathrm{Graal}^{3}$ confirment cette ambivalence. Il existe effectivement deux traditions au sein de ce vaste corpus composé d'une vingtaine d'ouvrages. La première est dite "chevaleresque » (FRAPPIER, 1977, p. 102) et retrace les aventures d'un chevalier en quête d'amour et d'exploits guerriers pour s'élever socialement ${ }^{4}$. La seconde est dite « ecclésiastique » (FRAPPIER, 1977, p. 103). La chevalerie est toujours glorifiée dans l'espace de la fiction mais les récits arthuriens revendiquent une

1 Jean-Louis Benoit interroge tout particulièrement la littérature médiévale à ce sujet (2006, p. 303-319).

2 Ces considérations ne sont pas sans rappeler les différents styles bibliques et homériques étudiés par Erich Auerbach (1968, p. 20-21).

3 Les romans du Graal représentent une vingtaine d'œuvres écrites en ancien français entre le $\mathrm{XII}^{\mathrm{e}}$ et le XIII ${ }^{\mathrm{e}}$ siècles. Ils relatent des aventures chevaleresques qui s'ancrent toujours plus ou moins dans l'histoire de Graal. Les chevaliers partent généralement en quête du saint Vessel après que celui-ci apparaît à la cour arthurienne.

4 Cette hypothèse de lecture est proposée par un certain nombre de critiques comme Jean Marx (1965), William Nitze (1999), Douglas Kelly (1993, p. 20) ou encore Jean Frappier (1977, p. 102-103). 
nouvelle forme de vérité. Par leur écriture en prose $^{5}$ et leur ancrage biblique, ils offrent désormais un "témoignage complet de la réalité eucharistique et de la grâce » et une " histoire sainte de la chevalerie » (FRAPPIER, 1977, p. 102 et p. 108). Nous pouvons alors parler de Hauts Livres du Graal ${ }^{6}$. Emblématique de cette démarche, ce plat mystérieux, qui passe et repasse devant Perceval dans Le Conte du Graal de Chrétien de Troyes, devient le Saint Vessel où Joseph d'Arimathie aurait recueilli le sang du Christ. Un pont se fait du roman à la Bible et réciproquement. La figure biblique qui offre son propre tombeau au corps du Christ devient le gardien du Graal, relique christique qui lui permet d'évangéliser les abords de Jérusalem puis les terres anglo-saxonnes. Le personnage et son Graal instaurent de la sorte une translatio ${ }^{7}$ 'Orient en Occident mais aussi de la Bible au roman. Ils assurent une continuité entre les Écritures et le Graal qui acquiert une origine biblique. De fait, le renouveau du corpus graalien se révèle par le biais de la présence d'une figure biblique qui devient un personnage de fiction à part entière dans l'espace romanesque. C'est cette interaction entre la Bible et

5 Le succès des romans du Graal se fait en même temps que l'essor de la prose. "Plus que le langage de la littérature religieuse, [la prose] est celui de la Bible, et plus que le langage de la Bible, celui de Dieu. Un livre qui renferme une révélation des desseins de Dieu doit être en prose. C'est le cas des romans du Graal, dès lors qu'ils retracent l'histoire familiale des gardiens du vase mystique, de Joseph d'Arimathie à Galaad ou à Perceval, dès lors que cette histoire est supposée intéresser le salut de l'humanité tout entière et reçoit un sens eschatologique lié au mystère de la Rédemption, dès lors enfin qu'elle se développe autour d'une sorte de noyau à la fois plein et vide, les paroles ultimes de la révélation divine, toujours dissimulées et toujours efficaces » (ZINK, 2006, p. 185). Une œuvre fait exception, le Roman de l'Estoire dou Graal de Robert de Boron. Cependant, initialement rédigée en vers, celle-ci a très rapidement été transposée en prose dans une version communément appelée Joseph d'Arimathie. Les deux versions sont réunies dans l'édition de Richard O'Gorman (Robert de Boron, 2005).

6 Jean-René Valette identifie ainsi ces œuvres. Selon lui, «à travers les romans de Robert de Boron, le Perlesvaus, la Queste ou l'Estoire del Saint Graal, il s'agit d'en appeler à une visée d'ordre religieux, de transmettre une foi, de communiquer ce que l'Estoire del Saint Graal nomme [...], "la grant hauteche precieuse de la sainte creanche au Sauveour vrai" (\$350)» (2015, p. 270-271).

7 Michel Stanesco propose une étude synthétique sur l'histoire du terme (2004, p. 467-477). Présente dès l'Antiquité, l'idée de translatio désigne la transmission d'un héritage culturel d'une civilisation à une autre. Au Moyen Âge, elle se fait de l'Orient vers l'Occident et, tout particulièrement pour nos romans, de la Palestine à la Grande-Bretagne. Dès le Cligès de Chrétien de Troyes, il est question de translation imperii et studii car « la chevalerie et la clergie sont passées d'Athènes à Rome, puis de Rome en France ». Néanmoins, pour nos romans, "l'idée de la translatio est située uniquement dans le cadre d'une économie providentielle et salvatrice » (ibid., p. 468). 
la littérature que nous nous proposons d'étudier en montrant comment la présence du personnage permet de fonder une nouvelle prose graalienne qui assume une pensée et une écriture bibliques.

Pour répondre à cette question, nous avons envisagé une étude de cas portant sur le statut du personnage. L'évolution de ce dernier témoigne effectivement non seulement de l'ancrage biblique des romans du Graal mais aussi de son dépassement. Nous verrons ainsi comment le décurion néotestamentaire se mue en saint chevalier, en personnage de fiction donc, tout en montrant comment il procure, malgré ce changement, des fondements scripturaires à la littérature graalienne.

\section{Le décurion biblique}

Joseph d'Arimathie suppose un intertexte biblique dans les œuvres où il apparaît. Comme tout personnage historique, il possède une "étiquette sémantique » (HAMON, 1977, p. 1428) préétablie dans un hypotexte voire dans une culture commune. Dans son cas, la revendication d'une filiation biblique implique alors qu'il y ait « seulement réactivation de certains attributs (physiques, vestimentaires, ou traits de caractère) » (DÉRUELLE, 2005, p. 98) déjà présents dans les versets évangéliques. Le lecteur doit être en mesure de reconnaittre à travers lui les traits distinctifs de la figure néotestamentaire. À l'inverse, si ce cahier des charges n'est pas respecté, le legs est contestable voire nié. De fait, les auteurs peuvent hésiter entre un respect des sources et une volonté de s'en émanciper au profit d'une dérive fictionnelle accordant davantage de place au Graal et aux quêtes que ce dernier engendre. Les romans de notre corpus mettent donc plus ou moins en avant les attributs et l'histoire de Joseph. Ce dernier constitue dès lors un marqueur révélant la nature et les degrés des liens que les œuvres entretiennent avec la Bible. Son étude permettra de déterminer si la prose graalienne assume une continuité ou non avec celle-ci.

8 «Le personnage est représenté, pris en charge et désigné sur la scène du texte par un signifiant discontinu, un ensemble de marques que l'on pourrait appeler "étiquette" dont les caractéristiques sont choisies par l'auteur» (HAMON, 1977, p. 142). Ces marques sont des «traits distinctifs». Désignant le signifié, l'unité de signification du personnage, «l'étiquette sémantique du personnage n'est pas une donnée a priori, et stable, qu'il s'agirait purement de reconnaître, mais une construction qui s'effectue progressivement, le temps d'une lecture » (ibid., p. 126). La reconnaissance du personnage biblique se fait donc au fil du texte et non en une seule fois à la faveur d'un portrait par exemple. 
Une approche comparative s'impose pour mieux considérer la variété de l'empreinte biblique dans nos textes. Ce n'est qu'en étudiant d'abord Joseph d'Arimathie à la source que nous pourrons déterminer ensuite comment les réécritures permettent de "penser le sacré » autrement mais toujours en «semblance » des Écritures?.

\section{Les textes bibliques et ecclésiastiques}

La Vulgate présente Joseph d'Arimathie comme « un décurion bon et juste [decurio vir bonus et justus] » (Luc 23, 50) voire comme un «noble décurion [nobilis decurio] qui attendait le règne de Dieu » (Marc 15, 42). Ne renvoyant à aucune réalité médiévale, ce statut n’est pas évident pour un lectorat de l'époque. De nombreux commentateurs ressentent alors le besoin de définir le terme pour éviter toute incompréhension ou tout malentendu. Dès le VII ${ }^{\mathrm{e}}$ siècle, Bède le Vénérable explique le verset de Luc ainsi :

Et voici qu'arriva un homme du nom de Joseph, qui était décurion, un homme bon et juste [decurio, vir bonus et justus]. Il n'avait pas appronvé leur décision et leurs actes. Il est appelé décurion parce que, dit-on, il est $<$ membre $>$ de l'ordre de la curie [ordine curiae] et dirige le service de la curie [officium curiae] ; il a aussi coutume d'être appelé « membre de la curie » [curialis] du fait qu'il assure des fonctions civiles (P.L., vol. 92, col. 0620D, nous traduisons).

L'épanode et l'accumulation des relatives révèlent la portée explicative du commentaire qui empêche tout contre-sens que les différentes acceptions du mot decurio pourraient causer. Ce dernier peut effectivement désigner un membre de la Curie, un conseiller donc, mais aussi le chef d'une décurie, d'un groupe de dix cavaliers. De fait, afin d'éviter toute confusion, Bède use de répétitions et précise avec lourdeur que la figure biblique a une fonction à la Curie et qu'il est également nommé « curialis », autrement dit membre d'une curie. Au fil des siècles, cette explication sera reprise presque mot pour mot par la plupart des auteurs chrétiens de Raban Maur au IX siècle à Zacharie de Besançon au XII ${ }^{\mathrm{e}}$ siècle ${ }^{10}$. Il est donc convenu, comme aujourd'hui, que

9 «Dès Robert de Boron, dont le texte emprunte beaucoup à la symbolique chrétienne tout en marquant soigneusement ses distances, le graal était devenu un signe, une "semblance", forcément imparfaite, des mystères divins » (BAUMGARTNER, 1999, p. 116).

10 En effet, selon Raban Maur, « Joseph est appelé décurion car il est de l’ordre de la Curie [ordine curiae] et il administre le service de la curie [officium curiae] » (P.L., vol. 107, col. 1146A, nous traduisons). Zacharie de Besançon procède de même (P.L., vol. 186, Col. 0586D). 
Joseph est un « membre notable du Conseil » (Marc 15, 42 et Luc 23, 50 ${ }^{11}$ ), autrement dit du Sanhédrin qui veillait au respect de la Loi juive. Certains auteurs prolongent néanmoins leur réflexion en s'aidant du grec. $\mathrm{Au} \mathrm{IX} \mathrm{Ix}^{\mathrm{e}}$ siècle, Paschase Radbert explique dans son commentaire de l'évangile de Matthieu que Joseph :

était un décurion, c'est-à-dire <un membre> de l'ordre de la curie [ordine curiae] dont il dirigeait le service, d'où le fait qu'il était aussi nommé «membre de la curie » [curialis], c'est-à-dire sénateur de la curie [senator curiae], que

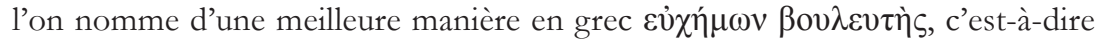
conseiller [consiliarius], parce qu'il s'occupait des conseils civils et publics [consilia civilia et publica] (P. L., vol. 120, col. 0971A, nous traduisons)

L'auteur utilise les mêmes procédés que Bède mais précise également que le terme decurio constitue une traduction trompeuse du grec qui identifie Joseph à un simple « conseiller notable».

De tels commentaires prouvent que le mot reste ambigu ce qui ne sera pas sans conséquence sur les traductions ou les adaptations bibliques qui sont faites en ancien français à partir du $\mathrm{XII}^{\mathrm{e}}$ siècle. Répondant avant tout à une volonté « didactique » (LECLERCQ, 1979, p. 273) d'éclairer les Écritures, ces textes révèlent différents procédés permettant de contourner l'ambiguïté lexicale du terme. À titre d'exemple, dans le manuscrit français 160, Joseph n'est que « noble » (Luc 23) ou «nobles hons et estoit venu de court » (Marc 15). Deux partis pris de traduction apparaissent. Dans le premier cas, l'auteur remplace le terme decurio, qui n'a pas d'équivalent en langue français, par un terme neutre qui atténue son sens. Dans le deuxième cas, il utilise une périphrase explicative. Notons alors qu'une certaine adaptation est déjà visible. Joseph devient non plus un membre de la Curie mais un homme venant de la cour.

Ce type d'infléchissement sémantique n'est pas rare dans les adaptations bibliques qui répondent à une démarche non seulement didactique mais aussi actualisante. Les auteurs réécrivent en effet les textes scripturaires en en proposant une traduction facile à comprendre et en les mettant en lien avec des réalités de leur temps. Tout est fait pour que le lecteur, quel qu'il soit, puisse accéder aisément mais correctement à la parole des Évangiles. Geufroy de Paris en offre un bon exemple en présentant Joseph dans la Bible des sept états du monde:

11 Nous renvoyons à La Bible de Jérusalem ou encore à la Traduction xcuménique de la Bible qui le désignent comme un «membre éminent du conseil » (Mc 15, 43 et Lc 23, 50). 
Uns hons estoit en la cité

Qui fu venu d'autre reigné,

Chevaliers estoit moult vaillanz,

Pilate avoit servi lonc tens.

Cil estoit Joseph apelez,

Sages iert et bien porpensez.

Venu li estoit en courage

Que il feroit grant vasselage

Qui le propheite qu'il ont mort,

Par grant envie et grant tort,

Metroit en soen monument

Ou il fust moult honestement (La Passion des jongleurs ${ }^{12}$, v. 3119-3130).

L'auteur cite et glose la Bible en évitant toute ambiguïté. Le « courage » de Joseph est explicitement mentionné mais aussi associé au « vasselage ». Il est également justifié grâce à la légitimité de sa requête qui résulte de ses bons services rendus à Pilate. Le vocabulaire est de nouveau simple et rappelle les valeurs chevaleresques des personnages de roman. Les termes chevalier, courage, vasselage ou encore vaillanz démontrent l'appropriation du discours biblique dans lequel apparaissent des références littéraires ou des réalités connues et donc plus facilement compréhensibles ${ }^{13}$. En somme, il s'agit de sélectionner ce qui est le plus important et le plus clair pour un lectorat non érudit sans pour autant porter atteinte aux sources ${ }^{14}$. Les adaptations instaurent d'ores et déjà des ponts entre la Bible et le monde médiéval. Elles confirment les conclusions de Jean Leclercq :

12 La Bible des sept états du monde que nous citons se trouvent dans La Passion des jongleurs. Texte établi d'après la Bible des sept estaz du monde de Geufroy de Paris (éd. Anne Joubert Amari Perry, 1981).

13 Macé de la Charité procède de même en présentant ainsi Joseph « qui de la cort de Pilate estoit » : «Cis Joseph tot celeement/ S'en vint au soir, mont humblement,/ A Pilate, que il servoit/ Et ja lonc tens il servi l'avoit,/Et li pria, s'il l'aime point,/ Que il le cors Jhesu li doint » (MACÉ DE LA CHARITÉ, p. 229, v. 3071430718).

14 Ces remarques ne concernent pas uniquement les romans du Graal. Elles révèlent plutôt une pratique de l'époque. Les mêmes problématiques et contraintes s'observent, par exemple, dans les romans antiques du Moyen Âge qui adaptent leurs sources selon des méthodes de suppression, de réduction, de synthèse et de substitution. Certains personnages antiques deviennent anonymes, les dieux sont remplacés par des sentiments, etc. Nous renvoyons à Aimé Petit, Naissance du roman. Les techniques littéraires dans les romans antiques du XII siècle, Genève, Slatkine, 1985, 2 vol. 
Au lieu d'interroger la Bible objectivement, on l'a interprétée, utilisée, pliée aux besoins de l'actualité. On y a fait un choix, puis on a appliqué les éléments retenus aux fins que l'on se proposait, à la façon dont on concevait la réforme et le pouvoir auquel il incombait de la réaliser (1979b, p. 103).

Toutes les traductions et toutes les gloses supposent donc une certaine « adhésion idéologique »(SALVADOR, 2007, p. 114). Ces observations révèlent d'ores et déjà comment les auteurs des romans du Graal étaient en moyen de se réapproprier le discours biblique non seulement en le respectant mais aussi en l'actualisant. Ces hypothèses de lecture restent néanmoins à vérifier dans l'espace graalien.

\section{Les romans du Graal}

Robert de Boron est le premier auteur à décrire Joseph d'Arimathie dans le Roman de l'estoire dou Graal:

A lui servoit uns soudoiers

Qui souz lui eut cinc chevaliers,

Jhesu Crist vit, et en sen cuer

L'aama mout ; meis a nul fuer

N'en osast feire nul semblant

Pour les Juïs qu'il doutoit tant,

Car tout estoient adversaire

A Jhesu la gent de pute eire.

Ainsi doutoit ses ennemis,

Ja soit ce qu’a Dieu fust amis (Joseph, éd. O’Gorman, v. 199-208).

Le substantif decurio est traduit par le nom soudoier qui renvoie à la deuxième acception du terme latin. Le vers suivant le confirme tout en précisant voire infléchissant le sens du mot. Joseph n'est pas n'importe quel soudoier, c'està-dire soldat ou homme d'armes travaillant à la solde de quelqu'un en échange d'une récompense. Il commande cinq chevaliers. Autrement dit, il a en charge une décurie. Ce changement de sens est acceptable mais a des conséquences sur la suite du récit qui fera explicitement de Joseph un chevalier. La retranscription en prose du passage laisse d'ailleurs déjà entendre une telle possibilité. Y est effectivement précisé que Pilate « avoit un sien chevalier soudoier a cinc chevaliers » (Joseph, éd. Richard O’Gorman, 1. 4041). La réunion des termes « chevalier » et « soudoier » révèle toute l'ambiguïté d'un geste de traduction qui 
oscille entre l'adaptation et le respect des sources. Cette hésitation ${ }^{15}$ n'apparait cependant plus lorsque Robert de Boron décroche de la trame narrative des Évangiles. L'auteur résout en effet de manière originale le problème que pose la polysémie du terme decurio. Au lieu de choisir telle ou telle acception du mot, il met à profit son potentiel sémantique en réunissant et hiérarchisant ses différentes définitions et donc traductions. Pilate témoigne face aux Juifs et présente Joseph comme "un soudoier, preudomme et mout boen chevalier » qui le servait «tout adés a cinc chevaliers a beles armes, a detriers » (Joseph, éd. Richard O'Gorman, v. 1351-1358). Le triptyque soudoierprodomme-chevalier confirme un respect des sources. Tous les désignatifs bibliques et apocryphes ${ }^{16}$ sont présents ${ }^{17}$. Cependant, une chronologie s'impose également entre les termes et suggère une évolution du personnage de la Bible au roman mais aussi du début du récit à sa fin. L'œuvre donne effectivement à voir comment le « soudoier » des Évangiles devient progressivement un chevalier après avoir été à la solde de Pilate. Une telle perspective donne sens au terme décurion en dépassant ses ambiguïtés et en légitimant dans le même temps la prose graalienne qui acquiert une filiation biblique par le biais de son récit mais aussi de son écriture. Robert de Boron pose ainsi, durablement, les fondements et la portée de l'histoire de Joseph d'Arimathie dans le corpus graalien.

Les œuvres ultérieures se pensent effectivement en continuité avec le Joseph d'Arimathie. Elles abandonnent progressivement les termes soudoier et

15 La version en prose se montre soucieuse de préciser ses choix de traduction en mentionnant que Pilate avait « un sien chevalier soudoier qui avoit non joseph et servait Pilate » « qui lavoit servi lonc tanc atout .v. chevaliers » et " dont li contes parlera asses » (texte et notes p. 43). L'assez grand nombre de variantes prouve que le statut de Joseph pose toujours un problème de traduction. Elles sont, d'ailleurs, moins nombreuses pour les autres qualificatifs donnés à Joseph.

16 Les apocryphes sont les textes qui témoignent des premiers temps de la chrétienté mais qui ont été rejetés des canons (Le mystère apocryphe, 1995). Certains sont très connus voire utilisés au Moyen Âge. Tel est le cas des deux apocryphes latins qui retracent les faits et gestes de Joseph d'Arimathie après la mise au Tombeau, L'Évangile de Nicodème (1973) et La Vengeance Nostre-Seigneur (1984). Le premier raconte comment le décurion est emprisonné par les Juifs puis libéré par des anges afin qu'il puisse témoigner de la Passion. La seconde relate comment il a participé à la vengeance de Vespasien contre les Juifs lors à Jérusalem. Les romans du Graal et tout particulièrement le Joseph d'Arimathie doivent beaucoup à ces ouvrages mais l'étude approfondie de ces derniers déborderait des limites de cet article puisqu'elle ne concerne pas directement une filiation avec la Bible.

17 Les adaptations font plutôt de Joseph un chevalier ou un soudoier, l'Évangile de Nicodème un prodome (tradition A, 1. 321 et tradition B, 1. 228) et la Vengeance Nostre-Seigneur un «saint chevaliers » (éd. Alvin E. Ford, p. 129). 
prodom quitte même à les omettre complétement. Tel est le cas de l'Estoire del Saint Graal en ce qui concerne le deuxième substantif. Dans les Continuations, Joseph est « li gentius cevaliers »(Première Continuation, 1993, v. 7534) " qui molt bons chevaliers estoit» ou « li prodom» ou encore « li vaillanz chevaliers » (First Continuation, vol II, 1949, v. 4689, v. 4697 et v. 17602). Dans le Lancelot-propre, la Dame du Lac, mère nourricière de Lancelot, enseigne à ce dernier les valeurs de la chevalerie et en vient, à sa demande, à citer les chevaliers qui les possédaient toutes. Joseph d'Arimathie, « le gentils chevaliers qui tant ama Jhesu Crist» (Lancelot, vol. 2, LX, 16), en fait évidemment partie (Lancelot, vol. 7, XXIa, 19). Dans la Queste del saint Graal, l'auteur parle de "Joseph d'Arimacie, li gentix chevaliers qui despendi Nostre Seignor de la sainte veraie Croix » ou encore de «Joseph d'Abarimacie li preudons, li verais chevaliers, [qui a quitté] Jherusalem par le commandement Nostre Seignor por preechier et por annoncier la verité de la Novele Loi et les comandementz de l'Evangile » (Queste, p. 32 et p. $134^{18}$ ). Au fil des œuvres, l'identité de Joseph se précise au gré de lourdes expansions du nom. Cellesci mettent progressivement en tension les termes prodom et chevalier tout en laissant entendre que le second découle du premier. Cette dialectique ne s'observe pas uniquement au sujet de Joseph ${ }^{19}$. L'ensemble des romans en prose instaure de plus en plus nettement une distinction entre les termes et la Queste représenterait d'ailleurs, selon Erich Köhler, l'« aboutissement d'une évolution qui commence dans les premiers romans de Chrétien ».

Joseph d'Arimathie apparaît [dans cette œuvre] comme « li preudoms, li verais chevaliers ». Seul le prodome est le vrai chevalier. Si la prodomie semblait être auparavant une conséquence naturelle de la chevalerie, cette même prodomie qui a désormais ses propres lois, déterminera à son tour la nature véritable de la chevalerie. L'évolution du concept moralisé de prodome vers un accomplissement spirituel de son sens lui donne de plus en plus une signification religieuse. À une époque où l'esprit bourgeois entreprend de s'approprier la prodomie, la noblesse s'attache justement au sens religieux de l'idéal chevaleresque (1974, p. 156).

18 La formule « li preudons, li verais chevaliers » a déjà été employée page 83.

19 Erich Köhler l'étudie dans le roman arthurien. Nous renvoyons à son article « Le concept de 'prodome' dans le roman arthurien, en particulier chez Chrétien de Troyes » (1974, p. 149-159). Ferdinand Lot s'est également intéressé au "verais chevalier». Selon lui, Joseph d'Arimathie et sa postérité représentent des " prototypes de la chevalerie » entendue comme « un idéal religieux et moral » qui « plonge ses racines jusqu'au début de l'humanité ». "L'orgueil de la prouesse individuelle, la gloire du siècle ne doivent jouer qu'un rôle secondaire dans la vie du vrai chevalier. Le but de son existence c'est la défense de Sainte Eglise, la protection des opprimés, des faibles, des femmes » (1984, p. 101). 
En d'autres termes, « la prodomie est l'expression suprême de la vie chevaleresque laïque, l'étape qui conduit à une chevalerie d'élite » (ibid., p. 158). Loin d'opposer des valeurs guerrières et morales, elle les rend compatibles et complémentaires. Celles-ci deviennent même indispensables à l'accomplissement d'un idéal chevaleresque. Joseph devient l'initiateur, le premier représentant, pour ne pas dire le créateur, d'une chevalerie d'excellence qui tire son origine au temps de la Passion ${ }^{20}$.

Une première étude lexicale des qualificatifs et des désignatifs renvoyant à Joseph d'Arimathie révèle à quel point la littérature graalienne assume une filiation avec la Bible. Le portrait du personnage et son évolution reposent sur un travail d'exégèse qui assure une continuité et une actualisation des Écritures. Les auteurs mettent à profit le potentiel des textes scripturaires afin de construire en toute légitimité leur récit. Dès lors, le décurion devient un chevalier qui assure le prestige de la chevalerie mise en scène dans les romans du Graal. Un tel constat reste cependant lacunaire. Dans l'immédiat, le terme chevalier n'est pas explicitement associé au Graal. Si nous nous en tenons aux mots, Joseph reste globalement perçu comme il l'était dans les adaptations bibliques. Notre première conclusion n'explique toujours pas comment, au niveau des récits graaliens et donc au-delà des Écritures, son histoire a pu autant se développer. Notre démarche lexicale doit être complétée par une recherche narratologique.

\section{Le chevalier du Graal}

Dans l'espace de la fiction, Joseph devient le chevalier qui a le Graal « an garde » (Joseph, éd. O’Gorman, 1. 324). La réappropriation du discours biblique dépasse largement la reprise telle quelle des traits distinctifs de la figure néotestamentaire. La question est alors de savoir si ce nouveau statut impose un écart total avec la Bible ou se pense plutôt comme une continuation fictive qui revendique malgré tout une empreinte biblique. Une telle interrogation suppose de considérer non plus les portraits de Joseph dont les caractéristiques sont reprises des Évangiles mais les récits qui le mettent en scène avec son Graal. Eux seuls permettent de voir plus clairement le degré d'émancipation de la fiction vis-à-vis des textes scripturaires.

20 Claude Lachet affirme qu'« en faisant de Joseph d'Arimathie, l'ami du Christ, un chevalier, c'est toute l'institution de la chevalerie que les écrivains transcendent et sanctifient. Au demeurant c'est au meilleur chevalier du monde, descendant de Joseph, qu'est réservé le Graal» (2006, p. 61). 
L'étude de l'histoire de Joseph révèlera comment certains procédés assurent une continuité narrative avec la Bible mais aussi un renouvellement du statut du personnage.

\section{Par et au-delà des Écritures : exégèse narrative et analogie}

Les Évangiles n'offrent pas assez de matière pour développer l'histoire de Joseph d'Arimathie. Seuls quelques traits distinctifs apparaissent finalement et les actes relatés apprennent avant tout sur la mise au Tombeau. Les auteurs ont donc dû trouver différents moyens pour donner consistance au personnage sans pour autant amoindrir l'ancrage biblique qu'il suppose. Deux procédés peuvent alors assurer plus ou moins directement cette filiation, l'exégèse narrative ${ }^{21}$ et l'analogie.

Une fois établie grâce aux commentaires, l'étiquette sémantique du personnage peut être mise en récit. Bien que fictive, l'histoire générée de la sorte assume un lien direct avec ses sources qu'elle dévoile juste différemment ${ }^{22}$. Dès lors, les romans ne se contentent plus de dire que Joseph est un sondoier qui a eu le courage de réclamer le corps du Christ. À l'instar des apocryphes, ils donnent à voir comment Joseph a répondu vertement aux Juifs :

[Les Juifs] avoient deffeit Jhesu.

Et quant Joseph l'a oï dire

Pleins fu de mautalent et d'ire,

Vint a Pilate isnelement

Et dist : «Servi t'ei longuement

Et je et $\mathrm{i}$ cinc chevalier,

N'en ei eü point de louier,

$\mathrm{Ne}$ ja n'en arei guerredon

Fors tant que me donras un don

De ce que touz jours prommis m'as.

Donne le moi, pouvoir en has. »

Pilate dist : «Or demandez, Je vous donrei ce que vourez.

Sanz la foiauté mon seigneur,

Nus ne l'aroit a mon honneur.

21 Ce procédé est observé dans les apocryphes (KAESTLI, 1995, p. 41). Il consiste à éclairer les Écritures par des récits qui les reprennent souvent ou, en tout cas, qui restent en accord avec leur potentiel sémantique dont témoignent les commentaires notamment.

22 En représentant des passages de la Bible, les Mystères et les Passions médiévaux procèdent de même mais par une mise en scène et non par une mise en récit. 
Vous avez granz dons deserviz.

- Sire, dist Joseph, granz merciz!

Je demant le cors de Jhesu,

Qu'il ont a tort en crouiz pendu. »

Pilates mout se merveilla

Quant si petit don demanda (Joseph, éd. O'Gorman, v. 438-458).

Ce passage dépasse largement la réécriture par amplification. L'auteur ne se contente pas de gonfler ses sources d'ajouts explicatifs. Il imagine plutôt le dialogue qu'auraient pu entretenir Pilate et Joseph. L'invention n'est cependant pas gratuite. Les caractéristiques de la figure biblique apparaissent clairement. Le personnage reste un décurion puisqu'il a servi Pilate avec ses cinq chevaliers. Il prouve par sa colère et la véhémence de son propos qu'il ne cautionne pas la décision des Juifs. Il affirme son courage face à eux. De fait, Robert de Boron reprend des méthodes d'écriture propres aux apocryphes et tout particulièrement à l'Évangile de Nicodème, d'ailleurs connu pour être l'une des sources principales de son œuvre ${ }^{23}$. Deux arguments le confirment. Joseph rencontre à plusieurs reprises les Juifs à qui il répond vertement ${ }^{24}$ (Écrits apocryphes chrétiens, 2005, t. 2, p. 279280) et la narration repose sur une " exégèse narrative » (KAESTLI, 1995, p. 41). Le ou les sens des Écritures sont effectivement dévoilés non plus par un commentaire explicite, une exégèse discursive, mais par un récit qui éclaire les Écritures en mettant en scène leur potentiel sémantique. Les traits distinctifs de Joseph sont traduits non plus en définitions mais en actes, les quelques versets des Évangiles devenant un dialogue qui les laisse transparaître.

Tout efficace qu'il soit, ce type d'invention s'avère difficile lorsque l'action relatée n'est aucunement présente dans les Évangiles ou dans un apocryphe. L'analogie permet alors de remédier au problème, de continuer le récit tout en restant en lien avec la Bible. Le procédé opère et se réitère à plusieurs reprises par transfert de personnage. Joseph agit comme d'autres figures bibliques et devient un modèle à suivre pour les membres de sa communauté. Ces derniers accomplissent et diffusent ensuite à leur tour le message divin en imitant leur guide et ainsi de suite.

23. Michel Zink étudie la question dans son article « Robert de Boron, la nature du Graal et la poétique du salut» (2003, p. 251303).

24 Les versions courtes de l'Évangile de Nicodème ne développent pas ce passage, se contentent de dire que Joseph réclame et obtient le corps. 
Conçue en écho avec l'Ancien et le Nouveau Testaments, l'histoire de Joseph fait parfois écho à celle d'un patriarche ${ }^{25}$ ou du Christ. Le personnage apparaît à bien des égards comme une figure moïsiaque. Dieu l'enjoint effectivement à construire une arche (Estoire, \60, 1. 18-23) pour y déposer le Graal grâce auquel il pourra lui parler et lui communiquer ses instructions pour la gouvernance de la compagnie. L'objet n'est pas sans rappeler l'Arche d'Alliance (Ex 21, 10-21) où sont déposés la « charte » et les dix commandements de Dieu. Les deux ouvrages connaissent par ailleurs un sort similaire. Ils suivent leur communauté jusqu'à être déposés dans un lieu de culte, le Temple de Salomon ou le Château du Graal où des moines séjournent. L'analogie légitime les actes de Joseph qui est perçu, à l'instar de Moïse, comme un gouverneur dépositaire des Lois de sa communauté. Une exégèse narrative apparaît à deux niveaux. D'une part, le récit narre comment Joseph d'Arimathie accomplit les paroles du Seigneur entendues par l'intermédiaire du Saint Vessel. D’autre part, ses actions réactualisent la réalisation des projets divins énoncés dans la Bible. Un écart s’impose néanmoins avec les Écritures et confirme l'hégémonie de la fiction. Le lien typologique instauré unit l'Ancien Testament non plus au Nouveau mais au roman qui devient en quelque sorte le récit d'une nouvelle alliance avec Dieu. Par ailleurs, l'exégèse narrative a perdu son ancrage biblique puisqu'elle émane de paroles fictives. Dès lors, seule la lecture typologique instaure réellement une empreinte biblique. La prose se joue sinon des codes d'écriture scripturaires pour s'accorder une légitimité trompeuse.

Les auteurs multiplient en conséquence les sources d'inspiration pour relier leurs histoires aux Écritures. Obéissant à une christomimesis (BUC, 1994, p. 28) propre au Moyen Âge ${ }^{26}$, Joseph accomplit également des actes analogues à ceux de Jésus ${ }^{27}$. Il est emprisonné par les Juifs dans une cellule qui rappelle le Tombeau. Il y reste par ailleurs trois ou quarante jours, périodes qui évoquent le temps entre la mort du Christ et la Résurrection ou la tentation

25 Madeleine Le Merrer montre que le personnage se construit à l'image de Joseph le patriarche notamment (1982, p. 229252).

26 Robert Javelet étudie également la question dans son introduction et son chapitre VI. "L'homme à l'image et à la ressemblance » (1967, vol.1, p. XIX-Xxilı et p. 169-245).

27 Joseph d'Arimathie est également une figure christique dans les apocryphes. Selon Rémi Gounelle, il est « un nouveau Jésus » car " presque tout ce qui arrive à Joseph d'Arimathée fait écho à ce qui est arrivé peu de temps auparavant à Jésus », "Joseph d’Arimathée, nouveau Jésus ? » (Évangile de Nicodème, 1973, p. 66-68). 
du Christ au désert (Marc 1, 13, Matthieu 4, 1-11, Luc 4, 1-13). Les Juifs tissent eux-mêmes des liens entre les deux personnages : ils affirment qu'ils redonneront le corps de Jésus uniquement si celui de Joseph leur est rendu. Dans le même ordre d'idée, les hommes de Nicodème découvrent les traces du décurion sur le Mont des Oliviers alors qu'ils cherchaient celles de Jésus. Enfin, tout comme ce dernier (Luc 19, 19-40), Joseph part pour Jérusalem sur un âne. Les analogies ne manquent pas pour en faire une figure christique. Ce rapprochement conduit à une «transvalorisation» (GOULLET, 2003, p. 113) du personnage qui gagne en consistance tout en conservant son prestige puisqu'il reste en lien avec la Bible. Il devient bel et bien un exemple à suivre pour sa communauté.

Ce statut particulier contribue à faire de lui la «pierre angulaire de la matière » (TRACHSLER, 2000, p. 14) de Judée $e^{28}$. Il fédère non seulement le récit mais aussi les personnages qui l'entourent. Preuve en est, dès la première œuvre de Robert de Boron, : l'action s'arrête lorsqu'il n'est plus question de lui. Le lecteur ne sait donc nullement ce qu'il advient durant son emprisonnement. La narration ne reprend que lorsqu'il est question de sa libération et que des Juifs en viennent à le rechercher. L'intérêt porte encore sur lui malgré son absence. De fait, à l'instar de certaines figures vétérotestamentaires, Joseph constitue un repère pour situer une époque historique et, plus techniquement, pour faire avancer le temps du récit. Il s'agit du «tans Joseph de Barimacie » (Roman de Tristan, tome VIII, \30, 1. 3233). À l'instar de Charlemagne ou d'Arthur (BOUTET, 1992), tout un univers se construit autour de lui. Ses compagnons s'adressent à lui au moindre problème et, tel un roi, il guide sa communauté selon les instructions divines. Le début de l'évangélisation des terres palestiniennes en témoigne :

Einsint vencha Vaspasiens le mort Jesucrist. Et quant Josep ot cele gent sauvee, si prist congié a Vespasien et s'en ala; en mout lointienes terres estranges les mena Josep. Et quant il furent [la], si lor moustra par maintes

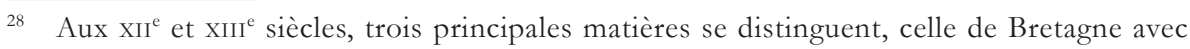
Arthur, celle de Rome avec Alexandre et celle de France avec Charlemagne. Chacune d'elles possède son personnage cadre, son espace et des projets d'écritures spécifiques. Nous renvoyons à l'ouvrage déjà cité de Richard Trachsler (2000) ainsi qu'à celui de Michel Zink (2006, p. 136 notamment). La matière de Judée désigne les textes dont le récit se déroule en Judée. Elle est aussi appelée par la critique "matière de Palestine ». Pour un bilan sur la notion et le rôle de Joseph, nous renvoyons à l'introduction de notre thèse Joseph d'Arimathie et les romans du Graal, dirigée par Jean-René Valette et soutenue à l'université Sorbonne Université en 2018. 
foïes et dist maintes beles paroles de nostre Seignor, si lor coumanda a labourer. Un mout grant piece ala lor afaire mout bien; et puis aprés si ala si mal con je vos conterai, que quanque il faisoient et labouroient aloit a mal. Et einsi furent une grant piece tant qu'il ne poaient plus soufrir. Et cil maus lor avenoit par une mauvese maniere de pechié que il avoient entr'aus coumanciee pour qoi tuit li bien terrien lor falloient; et cil pechiez estoit luxure sanz reson. Quant il furent si ataint que il ne porent plus soufrir, si vindrent a Ebron qui mout estoit bien de Josep (Joseph, éd. Richard O’Gorman 1. 954-964).

L'accomplissement de la vengeance de Vespasien montre que l'œuvre décroche des sources apocryphes. Le passage donne alors à voir le nouveau rôle attribué à Joseph. Moteur de l'action, ce dernier est d'abord le sujet de la plupart des verbes de la première partie du texte. Il guide sa communauté est tout se passe bien. L'ellipse temporelle laisse entendre que cette prospérité dure "mout grand piece » mais les événements prennent une nouvelle tournure et le récit change de forme. Joseph n'apparaît plus en position sujet et tout va mal. Ses compagnons se laissent tenter par le péché de luxure, engendrent la perte de leur communauté et en viennent à demander conseil à leur guide. Celui-ci s'affirme indubitablement comme une instance tutélaire. Il instaure l'ordre au sein de sa compagnie.

Du fait de son lien avec certaines figures bibliques et de son rôle fédérateur dans le récit, Joseph d'Arimathie devient un modèle à suivre qui permet de diffuser et perpétuer la révélation du Verbe tout au long du récit. Joseph est effectivement imité. Lorsqu'il s'agit de venger le Seigneur des Juifs, Vespasien décide d'abord d'exterminer les ennemis de Jésus mais tempère l'ardeur de sa vengeance sous l'influence de Joseph (Joseph, éd. Richard O'Gorman, p. 232-234). Par la suite, il en vient à son tour à chercher les messages laissés par Dieu sur Terre en vue de les transmettre (Joseph, éd. Richard O'Gorman, p. 228). Il s'engage ainsi, tout comme Joseph et Petrus $^{29}$, dans une mission évangélique. Alain, le neveu de Joseph, acquiert la " seignorie » de ses frères (Joseph, éd. Richard O'Gorman, v. 3184), à l'instar de son oncle qui assure la gouvernance de sa compagnie. Mieux encore, dans l'Estoire, Joseph donne naissance à un fils, Joséphé, qui contribue à la pérennité de son œuvre puisque Dieu lui-même lui accordera la charge du

29 Dieu soutient effectivement : «Je ne vueil qu'il y demeurt plus,/ Car vraiement dire pourra/ Que il seisi veü avra/ Hebron le Riche Pescheeur,/ Et dou veissel et de l'onneur » (Joseph, éd. Richard O'Gorman, v. 33853388). 
Graal et de sa communauté. Joseph assure de nouveau une empreinte biblique à la faveur de sa poétique. À l'instar d'autres figures bibliques étudiées par Philippe Lefebvre, il donne à voir comment

un personnage - secondaire ou pas - est $[\ldots]$ associé à des congénères qui partagent telle ou telle de ses expériences. Il tend à sortir de son site textuel et, éventuellement, de sa vie éphémère pour rejoindre une fratrie (LEFEBVRE, 2015, p. 12).

Nous retrouvons l'idée que Joseph est l'initiateur d'une chevalerie d'excellence conçue à son image.

En somme, il constitue un personnage cadre pour le récit : il génère l'action principale et représente un modèle à suivre pour ses compagnons. À la faveur de l'attitude exemplaire qu'il inspire aux autres, il diffuse le message biblique dans l'ensemble de l'œuvre. Conçus à son image, les personnages de la communauté du Graal imprègnent une aura biblique à la prose graalienne. Il reste néanmoins à dégager l'originalité de Joseph au sein de sa «fratrie ». En effet, comme le souligne Philippe Lefebvre,

par comparaison avec ses confrères, le personnage fugace laisse parfois entrevoir un trait qui le caractérise, le singularise, lui donne en d'autres termes une vraie teneur de personnage (LEFEBVRE, 2015, p. 10).

L'évolution du statut de Joseph demeure alors le marqueur le plus évident de sa singularité.

\section{Un saint laïque}

Dès que le roman ne réécrit plus directement la Bible mais s'en inspire, dès que Joseph foule les terres anglosaxonnes, il ne peut plus être pleinement associé à un patriarche ou à apôtre, figures qui ne renvoient plus aux réalités du XIII ${ }^{\mathrm{e}}$ siècle. Assurément, il est conçu à l'image de ces personnages bibliques mais en aucun cas il peut s'assimiler à eux car, une fois en Grande-Bretagne, les temps ont changé. Joseph a accompli sa première mission évangélique, celle qui est présente dans les textes scripturaires et apocryphes, et en vient à découvrir un territoire qui préfigure le monde arthurien. L'environnement perd en exotisme et rappelle davantage le monde médiéval avec ses rois, ses châteaux et ses chevaliers. De fait, Joseph acquiert un statut plus adapté aux réalités des $\mathrm{XIII}^{\mathrm{e}}$ siècle afin de montrer comment, après les apôtres, l'Homme peut encore, 
avec les moyens et les pensées qui lui sont propres, répandre le message du Christ. La figure biblique accède à un nouvel état emblématique du renouveau évangélique de l'époque, la sainteté (VAUCHEZ, 1986, p. 6-55). La Quatrième Continuation, l'Estoire, le Lancelot et le Sone de Nansay font effectivement de Joseph un saint homme lorsqu'il est question de ses actions en Grande-Bretagne. Ce changement de lieu mais aussi de désignatif va de pair et suggère que le « verais chevalier » évolue encore en fonction des mentalités médiévales. De fait, l'étude du personnage permet de voir comment les auteurs ont pu proposer une définition de la sainteté en tenant compte des spécificités de leur temps.

Dans la Quatrième Continuation, Joseph est d'abord un ancien " soldoiers » "plains de cortoisie » (v. 10378-10379). Homme à la solde de Pilate, Joseph appartient à un monde de cour. Une fois la Manche traversée, il s'entoure « de bone gens et saintisme » (v. 10408) et convertit le territoire grâce à son Graal. Sans être nié, son statut chevaleresque est omis. Il ne devient pourtant un « sains hom » (v. 10439) que lors de son emprisonnement dans le château du roi Crudel. Le narrateur précise alors que l'ensemble des prisonniers a survécu sans boire ni manger. C'est donc lorsque Joseph fait l'objet d'un miracle qu'il devient un saint à part entière et qu'il est en conséquence désigné comme tel. Selon les croyances du XIII ${ }^{\mathrm{e}}$ siècle, le saint est effectivement un intercesseur entre le Ciel et la Terre ${ }^{30}$ qui joue un rôle de guérisseurs et de protecteur pour sa communauté ${ }^{31}$. Avec son Graal, Joseph assure pleinement ces caractéristiques. Il prive ses compagnons de faim et les guides en leur transmettant les instructions divines entendues le plus souvent en présence de la sainte relique.

L'Estoire del saint Graal le confirme en proposant un portrait analogue du personnage. Une fois ressuscité par son intermédiaire, le roi Agron présente son bienfaiteur de la sorte :

Seignor, veez ici le saint home qui despendi le filz Deu de la veraie croiz et Dex l'a envoié entre vos, por ce que il velt que vos soiez bautizié, car autrement ne poez vos eschaper de la pardurable mort d'enfer (Estoire, \$790) ${ }^{32}$.

30 Selon Peter Brown, le saint représente un « compagnon invisible », une praesentia, autrement dit une présence physique du sacré qui possède des potentia (guérison, exorcisme, etc.) (2012, p. 113 et suivantes).

31 Les caractéristiques des saints ont pu varier selon les époques. Nous reprenons les plus usuelles et celles qui correspondent au XIII ${ }^{\mathrm{e}}$ siècle (BARNAY, 2004 et VAUCHEZ, 1986).

32 Notons que Joseph est encore un « saint homme » dans le même épisode du Lancelot, t.2, LXI, \$28. En ce qui concerne ces épisodes, nos remarques valent tant pour l'Estoire que pour le Lancelot. 
La revendication du statut de sainteté apparaît encore après une série de miracles et se perçoit comme l'aboutissement d'une quête personnelle menant à Dieu. Le Ciel ne fait cependant pas oublier la Terre. Joseph conserve ses fonctions séculières et œuvre au nom de Dieu pour les Hommes. Son statut de chevalier n'est aucunement remis en question et il est saint sans être un menistre de Dieu. Contrairement à son fils, il n'est jamais explicitement désigné comme un homme d'église. Ces remarques révèlent d'ores et déjà l'originalité du personnage qui apparait comme un saint laïque ${ }^{33}$, statut encore rare au XIII ${ }^{\mathrm{e}}$ siècle car plutôt réservé aux clercs et plus particulièrement aux évêques. Seules les saintetés dynastiques faisaient jusque-là exception mais notre personnage montre que la situation tend à évoluer. À l'instar de Robert de Boron qui unissait le prodomme, le soudoier et le chevalier, nos auteurs procèdent par ajout de termes pour marquer une évolution et non forcément une mutation qui supposerait un changement complet d'état. Joseph ne délaisse pas un statut pour un autre. Le "saint home » serait le quatrième et dernier terme d'un cheminement spirituel lä̈que.

Sone de Nansay témoigne du même parti pris. L'œuvre relate comment la figure biblique parvient à s'améliorer non seulement socialement mais aussi spirituellement. D’un côté, Joseph devient le « sains hons » (v. 4563) fondateur du Château du Graal. Son « sains cors » (v. 4557) y repose et c'est lui qui y institue les treize premiers moines (v. 48334838). D’un autre côté, il devient un chevalier en Grande-Bretagne puis le roi de Logres (v. 4784). À son arrivée, «Uns chevaus fu apparilliés/ Et unes armes pour parer/ Et pour chevalier adouber » (v. 47424744) et il finit par être « roy couronné » (v. 4784) à la demande de ses barons. Dans les deux cas, il parvient à l'excellence et le texte en vient même à brouiller parfois les frontières entre ses deux fonctions. En effet, « Mout men[e] li rois sainte vie,/ Sainte glyse en est essauchie. » (v. 4795) Cette formule est d'autant plus importante qu'elle est répétée à chaque jalon du récit, lorsqu'il est question des actions thaumaturgiques du Graal (v. 4690), de la naissance de Joséphé (v. 4700), « vesques crestiiennés » " preudons de saintes vie » (v. 4696-4697) et de la traversée de la Manche (v. 4716). L'expression disparaît quand Joseph rencontre une païenne et se marie avec elle sans le consentement divin. Elle n'est réemployée qu'après la naissance d'Adam, le fils infirme né de cette union. Ce handicap est interprété comme une forme de punition ou de rachat. Le texte précise effectivement : "par Adam poés

33 Les saints laïques sont étudiés notamment par André Vauchez (1972, p. 13-53 et 2004, p. 177239). 
prouver/ C'on ne doit commant trespasser » (v. 4857-4858). De fait, Joseph ne redevient un bon roi qu'en se repentant et en menant « sainte vie » (v. 4795). L'auteur en conclut d'ailleurs qu'« en sa vie essaucha li fois » (v. 4832) juste avant de préciser qu'il a institué « treze moinnes » (v. 4834).

La figure royale ne redevient positive que lorsqu'elle exalte l'Église et agit au nom de la foi chrétienne. Elle garantit la sûreté des reliques et contribue, par sa force, à répandre le nom de Dieu. Toute son ambivalence apparaît. À l'instar de Jean-Pierre Albert, nous pouvons observer que, dès qu'il est question du Graal, le roi

règne par la volonté de Dieu. Sa fonction est bien celle d'un roi, et il a tous les mérites chevaleresques d'un chef de guerre. Mais il est aussi une sorte de prêtre, selon un rite qui, pour être chrétien, n'implique cependant pas la reconnaissance ou la participation de l'Église (ALBERT, 1990, p. 297-298) ${ }^{34}$.

De fait, Joseph campe la posture d'un saint roi œuvrant pour Dieu et ses sujets. Il donne à voir comment un homme peut exceller dans deux sphères distinctes mais non inconciliables.

Quelles que soient les œuvres, Joseph montre que « les saints actualisent la Bible, témoignent du sens de l'Écriture sainte en la vivant. Et les légendes le disent »(BARNAY, 2004, p. 87). Son exemple révèle que l'Homme parvient à l'excellence tant qu'il respecte les volontés divines, tant que, par ses actions, " la fois [est] mout essauchie » (Sone, v. 4700). La prose graalienne se distingue en revendiquant une idée encore émergente au XIII ${ }^{\mathrm{e}}$ siècle. Un laïque peut accéder à la sainteté et deux voies principales s'ouvrent à lui : « l'assistance aux pauvres et l'expérience mystique » (VAUCHEZ, 1986, p. 39). Ces deux voies rappellent sans conteste le parcours de Joseph d'Arimathie dans Sone de Nansay. Or, il convient de rappeler que l'aboutissement de l'évolution du personnage ne contredit pas ses origines. Faire de lui un saint revient à assumer un legs biblique, à faire du décurion latin un saint chevalier.

Au terme de cette étude lexicale et narrative, nombreux procédés se combinent pour faire de Joseph d'Arimathie l'initiateur d'une chevalerie d'excellence conçue à son image et donc imprégnée d'une aura biblique. Partant du récit de la Passion, les romans du Graal continuent à diffuser le

34 Cet ouvrage est d'autant plus intéressant que Jean-Pierre Albert y étudie les «virtuoses religieux », ces êtres qui, comme Joseph, utilisent leur charisme pour influencer l'Histoire et infléchir les institutions. Pour plus de précisions, nous renvoyons également à notre thèse Joseph d'Arimathie et les romans du Graal, op. cit. 
message du Christ en l'actualisant selon les nouvelles réalités littéraires et sociohistoriques de leur temps. La reprise des traits distinctifs de la figure biblique et leur mise en récit confirment une volonté de ne pas enfreindre les sources évangéliques et apocryphes liées à l'histoire du personnage. L'analogie et l'exégèse narrative révèlent quant à elles comment les œuvres s'élaborent en écho avec la Bible même lorsqu'elles s'éloignent de la trame originelle de l'histoire du décurion. Joseph devient bel et bien un marqueur de l'empreinte biblique de la prose graalienne.

\section{Bibliographie}

\section{Sources}

BÈDE LE VÉNÉRABLE, Lucea Evangelium expositio in Opera omnia Bedea, Liber sextus, Caput XXIII, P. L., vol. 92.

Bible historiale, Petite Bible historiale complétée, Paris, Bibliothèque nationale de France, Manuscrit Français 160, 1310.

Écrits apocryphes chrétiens, Paris, Gallimard, « Bibliothèque de la Pléiade », 2005.

Estoire del Saint Graal (L'), éd. Jean-Paul Ponceau, Paris, Champion, « CFMA », 1997, 2 vol.

First Continuation (The). The Continuations of the Old French Perceval of Chrétien de Troyes, ed. William Roach, Philadelphia, University of Pennsylvania Press (vol. I, II) / The American Philosophical Society (vol. III), 1949, 1950, 1952.

Évangile de Nicodème. Les versions courtes en ancien français et en prose (L'), éd. Alvin E. Ford, Genève, Droz, 1973.

Lancelot. Roman en prose du XIII ${ }^{\mathrm{e}}$ siècle, éd. Alexandre Micha, Genève, Droz, 19781983, 9 vol.

MACÉ DE LA CHARITÉ, La Bible de Macé La Charité, VI, Evangiles, Actes des Apostres, dir. Jean-Robert Smeets, Leiden, Leiden University Press, 1986.

Patrologiae cursus completus. Serie latina, éd. Jacques-Paul Migne, 217 vol. et 4 vol. de Tables, Paris, 1844-1865 (version numérisée, http://pld.chadwyck.com/).

PASCHASE RADBERT, Expositio in Evangelium Matthaei, in Opera omnia Sancti Paschasii Radberti, P. L., vol.120.

Passion des jongleurs (La). Texte établi d'après la Bible des sept estaz du monde de Geufroy de Paris, éd. Anne Joubert Amari Perry, Paris, Beauchesne, «Textes Dossiers Documents » 4, 1981.

Première continuation de Perceval (Continuation-Gauvain). Texte du ms L [version brève], éd. William Roach, trad. et prés. par ColetteAnne Van CoolputStorms, Paris, Le Livre de Poche, «Lettres Gothiques », 1993. 
Queste del Saint Graal (La). Roman du XIII' siècle, éd. Albert Pauphilet, Paris, Champion, «CFMA », 2003 [1984].

ROBERT DE BORON, Joseph d'Arimathie. A Critical Édition of the Verse and Prose Versions, ed. Richard O'Gorman, Toronto, Pontifical Institute of Mediaeval Studies, «Studies and Texts »120, 1995.

ROBERT DE BORON, Le Roman de l'Estoire dou Graal, éd. William Nitze, Paris, Champion, « CFMA », 1999 [1971].

Roman de Tristan en prose (Le), dir. Philippe Ménard, Genève, Droz, 1987-1997, 9 vol.

Sone de Nansay, éd. Claude Lachet, Paris, Champion, «CFMA », 2014.

Traduction œcuménique de la Bible, Paris, Éditions du Cerf, 2004.

Vengeance de Nostre-Seigneur. The Version of Japheth (La), éd. Alvin E. Ford, Toronto, Pontifical Institute of Mediaeval Studies, «Studies and Texts » 63, 1984.

\section{Études}

ALBERT, Jean-Pierre, Odeurs de sainteté. La mythologie chrétienne des aromates, Paris, Éditions de l'École des Hautes Études en Sciences Sociales, 1990.

AUERBACH, Erich, Mimésis. La représentation de la réalité dans la littérature occidentale, trad. Cornélius Heim, Paris, Gallimard, "Tel », 1968 [Mimesis : dargestellte Wirlichkeit in det abendländischen Literatur, 1946].

BARNAY, Sylvie, Les Saints. Des êtres de chair et de ciel, Paris, Gallimard, " Découvertes », 2004.

BAumgartner, Emmanuèle, Chrétien de Troyes. Le Conte du Graal, Paris, Puf, «Études littéraires », 1999.

BENOIT, Jean-Louis, « Clef du texte, clef du royaume. La lecture de la Bible au Moyen Âge comme paradigme de la littérature ", Les clefs des textes médiévaux. Pouvoir, savoir et interprétation, dir. Fabienne Pomel, Rennes, PUR, «Interférence », 2006, p. 303-319.

BROWN, Peter, Le culte des saints. Son essor et sa fonction dans la chrétienté latine, Paris, Cerf, « Biblis », 2012 [1984].

BOUTET, Dominique, Charlemagne et Arthur ou le roi imaginaire, Paris, Champion, 1992.

BUC, Philippe, L'ambiguïté du Livre/ Prince, pouvoir et peuple dans les commentaires de la Bible au Moyen âge, Paris, Beauchesne, 1994.

DÉRUELLE, Aude, «Le cas du personnage historique », L'Année balzacienne 2005/1 (nº). FRAPPIER, Jean, «Le Graal et la chevalerie » [1954], Autour du Graal, Genève, Librairie Droz, 1977.

FRYE, Northrop, Le Grand Code. La Bible et la littérature, préface de Tzvetan Todorov, trad. de l'anglais par Catherine Malamoud, Paris, Seuil, 1984 [The Great Code. The Bible and Literature, 1981, 1982]. 
HAMON, Philippe, «Pour un statut sémiologique du personnage », Poétique du récit, Paris, Seuil, « Points », 1977, p. 115-180.

GOULLET, Monique, «Vers une typologie des réécritures hagiographiques, à partir de quelques exemples du Nord-Est de la France. Avec une édition synoptique des deux Vies de saint Evre de Toul », La réécriture hagiographique dans l'Occident médiéval. Transformations formelles et idéologiques, dir. de Monique Goullet et Martin Heinzelmann, 2003, Ostfildern, J. Thorbecke, «Beihefte der Francia » 58, p.111146.

Histoire des Saints et de la sainteté chrétienne. Tome VI. Au temps du renouveau évangélique (1054-1274), dir. André Vauchez, Paris, Hachette, 1986.

JAVELET, Robert, Image et ressemblance au douzième siècle. De saint Anselme à Alain de Lille, Paris, Letouzé et Ané, 1967, 2 vol.

KAESTLI, Jean-Daniel, «Les écrits apocryphes chrétiens. Pour une approche qui valorise leur diversité et leurs attaches bibliques ", Le mystère apocryphe. Introduction à une littérature méconnue, dir. Jean-Daniel Kaestli et Daniel Marguerat, Genève, Labor et Fides, 1995 (2 $2^{\mathrm{ème}}$ édition), p. 29-44.

KELLY, Douglas, Medieval French Romance, New York/Toronto, « Twayne’s World Authors Series. French literature »838, New York/Toronto, 1993.

KÖHLER, Erich, « Le concept de 'prodome'» dans le roman arthurien, en particulier chez Chrétien de Troyes ", L’aventure chevaleresque, Idéal et réalité dans le roman courtois, trad. de l'allemand par Eliane Kaufholz, Paris, Gallimard coll. "Bibliothèque des idées ", 1974, p. 149-159.

LACHET, Claude, "La chevalerie au XIII ${ }^{e}$ siècle : ombres et lumières », Regards sur la chevalerie de l'Europe médiévale. Histoire et imaginaire, dir. Jean Dufournet et Francis Dubost, Revue des Langues Romanes, tome CX, n¹, 2006, p. 57-75.

LE MERRER, Madeleine, «Figure de Joseph d'Arimathie. Sa chasteté, sa proximité de Dieu », Images et signes de l'Orient dans 1'Occident médiéval, Senefiance, 11, 1982, p. 229252.

LECLERCQ, Jean, «Les traductions de la Bible et la spiritualité médiévale », The Bible and medieval culture, éd. Willem Lourdaux et Daniël Verhelst, Louvain, Presses Universitaires de Louvain, 1979, p. 263-278.

LECLERCQ, Jean, «Usage et abus de la Bible au temps de la réforme grégorienne », ibid., p. $89-108$.

LEFEBVRE, Philippe, Brèves rencontres. Vies minuscules de la Bible, Paris, Éditions du Cerf, 2015.

LOBRICHON, Guy, «Les traductions médiévales de la Bible dans l'Occident latin », Biblia.

Les Bibles en latin au temps des Réformes, dir. Marie-Christine Gomez-Géraud, Paris, Presses de l'université Paris-Sorbonne, coll. «Religions dans l'histoire », 2008, p. 9-36.

LOBRICHON, Guy, La Bible au Moyen Âge, Paris, Éditions A. et J. Picard, « Les médiévistes français », 3, 2003.

LOT, Ferdinand, Étude sur le Lancelot en prose, Paris, Champion, 1984. 
MARX, Jean, Nouvelles recherches sur la littérature arthurienne, Paris, Klincksieck, 1965.

Mystère apocryphe. Introduction à une littérature méconnue (Le), dir. Jean-Daniel Kaestli et Daniel Marguerat, Genève, Labor et Fides, 1995

SALVADOR, Xavier-Laurent, Vérité et écriture(s), Paris, Champion, 2007.

STANESCO, Michel, «La dernière frontière et la fin des temps », Frontières et senils, dir. Joëlle Ducos, Eidôlon, 67, 2004, p. 467-477.

TRACHSLER, Richard, Disjointures-Conjointures. Étude sur l'interférence des matières narratives dans la littérature française du Moyen Âge, Tübingen/Basel, A. Francke Verlag, «Romanica Helvetica »120, 2000.

VALETTE, Jean-René, «Les Hauts Livres du Graal et la poétique des genres. Éléments de définition ", Motifs merveilleux et poétique des genres au Moyen Âge, dir. Francis Gingras, Paris, Classiques Garnier, «Rencontres » 151, 2015, p. 267286.

VAUCHEZ, André, « De nouvelles saintetés ? Renouveau de la sainteté traditionnelle et apparition des saints laïques ", Les saints et l'Histoire. Sources hagiographiques du haut Moyen Âge, dir. Anne Wagner, Paris, Bréal, 2004, p. 177239.

VAUCHEZ, André, "Sainteté laïque au XIII siècle : la vie du bienheureux Facio Crémone (v. 1196-1272) », Mélanges de l'École française de Rome. Moyen-Age. Temps modernes, tome $84, \mathrm{n}^{\circ} 1,1972$, p. 1353.

VAUCHEZ, André, "La sainteté au temps du renouveau évangélique », Histoire des Saints et de la sainteté chrétienne. Tome VI. Au temps du renouveau évangélique..., op.cit., p. 6-55.

ZINK, Michel, Poésie et conversion, Paris, Puf, 2003. Voir en partic. le chap. « Robert de Boron, la nature du Graal et la poétique du salut », p. 251303.

ZINK, Michel, Littérature française du Moyen Âge, Paris, PUF, « Quadrige », 2006 [1992].

Submetido em: 24-10-2019

Aceito em: 21-11-2019 